\title{
Síndrome de congestión pélvica, una enfermedad subdiagnosticada
}

\author{
Roberto Aguila-Márquez, ${ }^{1}$ Liza Ochoa Armendáriz ${ }^{2}$
}

\section{INTRODUCCIÓN}

El dolor pélvico crónico se define como la presencia de dolor abdominal y de la región pélvica que dura más de seis meses. Puede originarse por múltiples causas; sin embargo, una de ellas, subdiagnosticada, es el síndrome de congestión pélvica, resultado del reflujo u obstrucción de las venas gonadales, principalmente. Este padecimiento representa el $30 \%$ de la consulta externa ginecológica en EUA y afecta hasta al $40 \%$ de la población femenina durante su vida, en un rango de edad de los 20 a los 45 años y en la premenopausia. ${ }^{1}$

Como es sabido, el drenaje venoso del útero y los ovarios es a través de las venas iliacas internas y gonadales. La vena ovárica izquierda drena en la vena renal izquierda y la vena ovárica derecha drena directamente a la vena cava inferior. Las venas ováricas normalmente miden menos de cinco milímetros de diámetro y poseen tres válvulas, principalmente en el tercio distal. Las válvulas pueden ser incompetentes hasta en el $40 \%{ }^{2}$

El síndrome de congestión pélvica (SCP) se caracteriza por dolor abdominal bajo o pélvico inespecífico, dismenorrea, disuria, dispareunia, sensación de pesadez en las piernas; comúnmente, la paciente presenta várices vulvares o paquetes varicosos en sitios anatómicos poco frecuentes en mujeres y varicocele de repetición en hombres. Las várices pélvicas pueden desarrollarse por dos mecanismos: reflujo causado por incompetencia valvular u obstrucción.

La causa de la incompetencia valvular se desconoce, aunque se cree que los factores hormonales juegan un

\footnotetext{
${ }^{1}$ Cirugía Vascular y Endovascular, Hospital Ángeles Lomas.

2 Cirugía Vascular y Endovascular, Centro Médico Nacional 20 de Noviembre, ISSSTE.
}

Correspondencia:

Roberto Aguila-Márquez

Correo electrónico: vasculara@yahoo.com.mx

Aceptado: 21-09-2016.

Este artículo puede ser consultado en versión completa en http:// www.medigraphic.com/actamedica papel significativo. La causa más frecuente de obstrucción del flujo de salida de la vena ovárica es la compresión de la vena renal izquierda entre la arteria mesentérica superior y la aorta, también conocido como "síndrome del cascanueces" (nutcracker syndrome). La obstrucción distal lleva a un aumento de la presión venosa y una dilatación venosa subsecuente, insuficiencia valvular y tortuosidad de la vena ovárica, lo que resulta en un gradiente de presión elevado entre la vena renal izquierda y la vena cava. ${ }^{1}$

En el examen físico pueden encontrarse várices vulvares, congestión del cuello uterino con dolor a la movilización, hematuria, várices en miembros inferiores (hasta en el $50 \%$ de los individuos), hemorroides, várices perineales y glúteas.

\section{ESTUDIOS DE IMAGEN}

Para el análisis en conjunto, el primer estudio deberá ser un ultrasonido pélvico para visualizar los vasos gonadales. Puede realizarse USG transabdominal y transvaginal. Un diámetro mayor de seis milímetros de las venas ováricas se considera patológico. El Doppler dúplex tiene una sensibilidad de $100 \%$ para la dilatación de la vena ovárica izquierda y de 67\% para la vena ovárica derecha. Deben ser valoradas las venas iliacas internas, comunes y renales en busca de obstrucción, así como las venas femorales en busca de reflujo. 3,4

La angiorresonancia magnética tiene mayor sensibilidad (91.7\%) y especificidad (88.9\%), particularmente en casos compresivos como May Thurner y el síndrome del Cascanueces. Este mismo estudio realizado en tiempo real es particularmente útil para la detección de SCP por su capacidad para determinar con exactitud el flujo anterógrado o retrógrado en la vena ovárica. La venografía es el estándar de oro para el diagnóstico de SCP; sin embargo, debe reservarse no sólo para el diagnóstico sino como herramienta en el tratamiento mínimamente invasivo.

\section{TRATAMIENTO ENDOVASCULAR: EMBOLIZACIÓN}

Se ha convertido en el pilar para el tratamiento del SCP secundario a incompetencia venosa pélvica y ovárica. Los 
agentes para embolización son la espuma esclerosante, pegamento (cianocrilato) y dispositivos de cierre u oclusores (como discos y espirales).

La tasa de éxito para la reducción o eliminación del dolor pélvico crónico es de hasta 90\% con seguimiento a cinco años. ${ }^{3,5}$

La embolización se puede realizar por acceso yugular o femoral; a través de la vena femoral, vena iliaca y vena cava, se canulan la vena renal izquierda y vena ovárica. Con el paciente en Trendelemburg invertido, se lleva a cabo un disparo de medio de contraste y se observa el reflujo hacia la pelvis, dilatación de la vena ovárica y presencia de colaterales. Estos dos datos son sumamente importantes y realizan el diagnóstico definitivo de la patología. La canulación del tronco principal de la vena ovárica puede facilitarse con microcatéteres; posteriormente, se liberan los dispositivos de cierre de distal a proximal, lo que favorece la trombosis de la vena. La complicación más grave es la migración de los dispositivos oclusores a la arteria pulmonar, ${ }^{6}$ que puede presentarse en venas de diámetro mayor a $12 \mathrm{~mm}$. Para

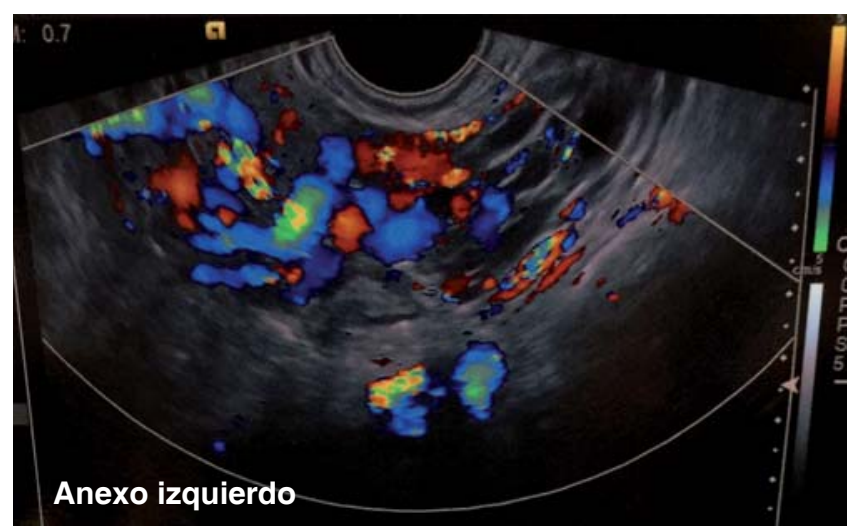

Figura 1. Ultrasonido Doppler transvaginal con presencia de venas ováricas mayores a $6 \mathrm{~mm}$, con aumento de velocidades $y$ flujo reverso de manera bilateral.
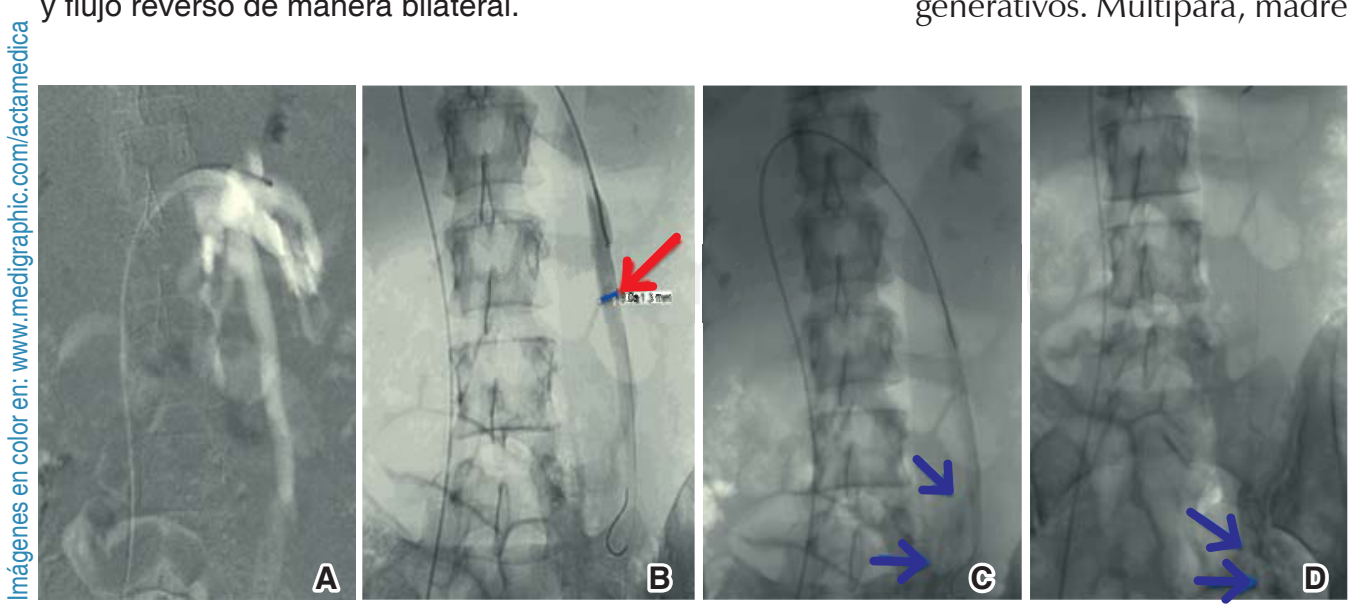

Figura 2.

Se observa vena renal izquierda canulada, con dilatación importante de la vena gonadal izquierda (A), con medición de 1.3 $\mathrm{mm}$ de diámetro en disparo selectivo (B). Múltiples venas colaterales dilatadas, ramas de la vena gonadal izquierda (flechas C y D). 
En el año 2012 se realizó safenoablación por radiofrecuencia por reflujo safeno femoral bilateral. Tres años tras la cirugía comenzó con aparición de várices en la región vulvar y en la extremidad inferior izquierda. Refería pesadez pélvica, dismenorrea e incapacidad para permanecer sentada por largos periodos por exacerbación de los síntomas, además de dispareunia ocasional.

Se realizó ultrasonido Doppler venoso, en donde se observaron venas safenas ocluidas por antecedente quirúrgico y vena intersafena izquierda permeable.

El ultrasonido Doppler transvaginal mostró presencia de venas ováricas mayores a seis milímetros, con aumento de velocidades y flujo reverso de manera bilateral (Figura 1).

Se llegó al diagnóstico de síndrome de congestión pélvica secundario a reflujo de venas ováricas y se decidió realizar un procedimiento endovascular en noviembre de 2015.

Se llevó a la mujer a la sala de hemodinamia; bajo anestesia general, se llevó a cabo punción de ambas venas
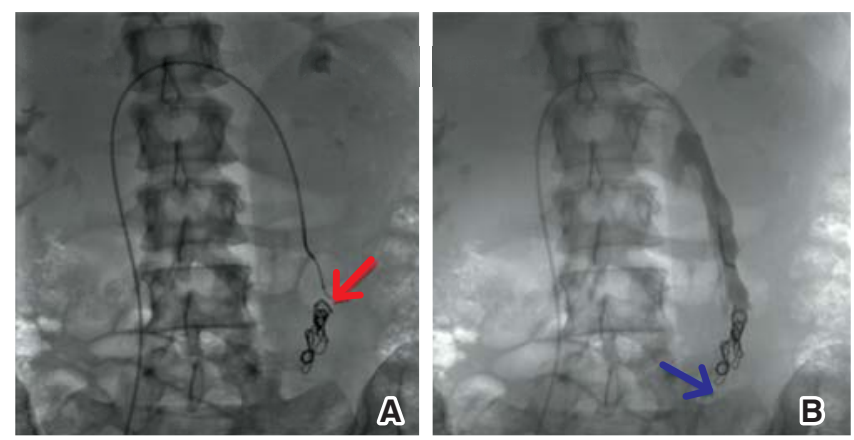

Figura 3. Embolización selectiva con dispositivos de cierre en espiral de la vena gonadal izquierda (A). Ausencia de colaterales dilatadas en disparo de control (B). femorales y se colocaron introductores 6 Fr. A través del acceso derecho, se canuló la vena cava inferior; posteriormente, la vena renal izquierda (Figura 2A). Se realizó un disparo con medio de contraste y se descartó causa oclusiva. Se visualizó la vena gonadal izquierda dilatada (Figura 2B). Con posición de Trendelemburg modificada, se observó importante reflujo y presencia de venas colaterales dilatadas en la pelvis (Figura 2C y 2D). Se canuló, de igual manera, la vena hipogástrica derecha con técnica de paso de guía contralateral a través del acceso izquierdo y se observaron abundantes colaterales y reflujo venoso. Se decidió realizar embolización selectiva con oclusores espirales, con desaparición evidente de las venas colaterales (Figuras 3 y 4 ).

En el postquirúrgico, la paciente evolucionó favorablemente, con desaparición completa de los paquetes varicosos a las dos semanas, así como remisión de la sintomatología. Actualmente niega dolor pélvico.

El síndrome de congestión pélvica representa un reto diagnóstico, por lo que debemos tener presentes las patologías asociadas, así como los métodos y criterios diagnósticos específicos para identificarlo correctamente, sin perder objetividad acerca de la enfermedad venosa de miembros inferiores o una sintomatología abdominal inespecífica que puede tener un trasfondo mayor. El tratamiento endovascular ha demostrado ser eficaz en la resolución de los síntomas según lo reportado en la literatura, además de ser un método mínimamente invasivo y con baja tasa de complicaciones; se deberá individualizar en cada sujeto la mejor alternativa terapéutica. En el caso presentado, los resultados obtenidos fueron satisfactorios, ya que la mujer refiere una mejoría en su calidad de vida, así como en el aspecto estético, con la desaparición de las venas varicosas en la región pélvica.
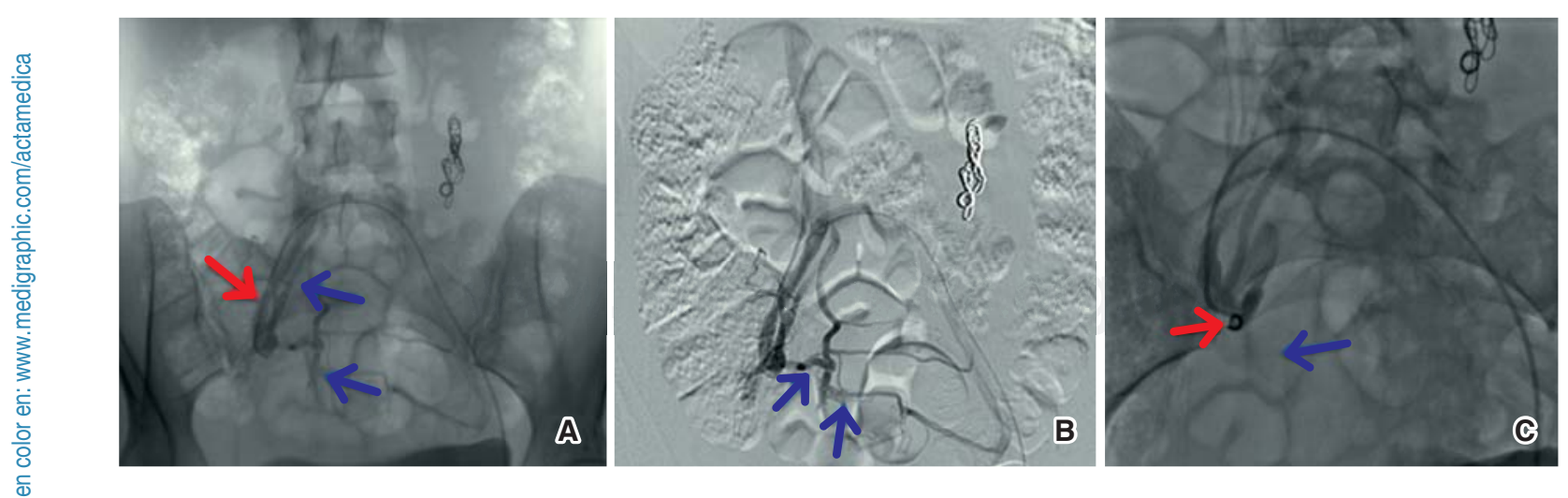

Figura 4. Canulación selectiva de la vena hipogástrica derecha (A. Flecha roja), se observan múltiples venas colaterales (A y B Flechas azules). Embolización selectiva con dispositivo de cierre en espiral del tronco venoso principal (C. Flecha roja), 監 desaparición de venas colaterales (C. Flecha azul). 


\section{REFERENCIAS}

1. O'Brien MT, Gillespie DL. Diagnosis and treatment of the pelvic congestion syndrome. J Vasc Surg Venous Lymphat Disord. 2015; 3: 96-106.

2. Kurklinsky AK, Rooke TW. Nutcracker phenomenon and nutcracker syndrome. Mayo Clin Proc. 2010; 85: 552-559.

3. Thors A, Haurani MJ, Gregio TK, Go MR. Endovascular intervention for pelvic congestion syndrome is justified for chronic pelvic pain relief and patient satisfaction. J Vasc Surg Venous Lymphat Disord. 2014; 2: 268-273.

4. Malgor RD, Adrahtas D, Spentzouris G, Gasparis AP, Tassiopoulos AK, Labropoulos N. The role of duplex ultrasound in the workup of pelvic congestion syndrome. J Vasc Surg Venous Lymphat Disord. 2014; 2: 34-38.

5. Mahmoud O, Vikatmaa P, Aho P, Halmesmäki K, Albäck A, RahkolaSoisalo $P$ et al. Efficacy of endovascular treatment for pelvic congestion syndrome. J Vasc Surg Venous Lymphat Disord. 2016; 4 (3): $355-370$.

6. Yamasaki W, Kakizawa H, Ishikawa M, Date S, Tatsugami F, Terada H et al. Migration to the pulmonary artery of nine metallic coils placed in the internal iliac vein for treatment of giant rectal varices. Acta Radiol Short Rep. 2012; 1 (6): arsr.2012.120024.

7. Liu Y, Sun Y, Wu XJ et al. Endovascular stent placement for the treatment of nutcracker syndrome. Int Urol Nephrol. 2012; 44: 1097.

8. Jayaraj A, Gloviczki P, Peeran S, Canton L et al. Hybrid intervention for treatment of the nutcracker syndrome. J Vasc Surg Cases. 2015; 1: 268-271

9. Wang X, Zhang Y, Li C, Zhang H. Results of endovascular treatment for patients with nutcracker syndrome. J Vasc Surg. 2012; 56: 142-148.

10. White JV, Ryjewski C, Messersmith RN, Sbrana F, Schwartz LB. Left ovarian to left external iliac vein transposition for the treatment of nutcracker syndrome. J Vasc Surg Venous Lymphat Disord. 2016; 4: 114-118. 